\title{
Industrial Automation in Unit Operations Labs
}

\author{
Konstantinos Apostolou and Ishwar Singh \\ School of Engineering Technology \\ McMaster University \\ Hamilton, Ontario, Canada \\ apostol@mcmaster.ca
}

\begin{abstract}
Chemical engineering unit operation labs do an excellent job of integrating the whole curriculum and exposing students to pilot-scale systems. Where they are often lacking, though, is the exposure to and use of real-life industrial automation by the future graduates. A unit operation lab that has been automated using industrial level paradigms and equipment is the focus of this paper. A partnership with a global automation manufacturer (Emerson) was established and the lab was retrofitted using industrial sensors and actuators, a Distributed Control System (DeltaV DCS), industrial networks (FOUNDATION Fieldbus and AS-i), Human Machine Interface (HMI) screens, and system redundancy. The details of the automation along with its use through the lab curriculum will be discussed. This cross-curricular approach benefits students as, through the regular unit operation labs, they become familiar with key elements of an automated set-up, understand the need for it and its limitations, see control loops in action, communicate to the units through the HMI, and use the HMI to recover historical data on the processes. The lab is a meso-scale of a processing facility and prepares students for field work after graduation. At the same time, the traditional exposure to "manually operated" sensors and final elements is maintained as some of the units have not been converted to fully automated systems.
\end{abstract}

Keywords: Chemical Engineering, unit operations, automation, DCS, laboratory, process management, DeltaV.

\section{INTRODUCTION}

Unit Operations labs are a core element in every Chemical Engineering curriculum. They integrate many elements of the curriculum (mass and energy balances, thermodynamics, heat transfer, fluid mechanics, process modeling, problem-solving, analysis, and communication) and are often the only place where students are exposed to pilot scale processes and get a flavour of industrial-like equipment. Whereas industrial processes have been for years governed by strict control principles and are monitored through modern Supervisory Control and Data
Acquisition (SCADA) programs [4], academic unit operation labs often lack industrial-type control elements. This is a major shortcoming, when the labs' pivotal role in connecting the curriculum to industrial practice is considered. Even when such control exists, it is often isolated on a single unit, as in [1], [3], and [5], and rarely offers the ability to monitor and control a complete set of processes, as in [6].

This paper presents the Distributed Control System (DCS) process management set-up at Mohawk College's Chemical Engineering lab in Hamilton, Ontario, Canada. It outlines the lab equipment, its instrumentation, the Distributed Control System (DCS), its use by the students, and the anticipated student benefits. Suggestions for improvement of this and other unit operations labs are included.

\section{LABORATORY DESCRIPTION}

The Chemical Engineering lab at Mohawk College is a versatile space equipped with a series of small-scale units and a number of fixed-position pilot-scale equipment. The small-scale units are utilized for, among others, experiments in tubular membrane ultra-filtration, metals precipitation, phosphate precipitation, waste-water neutralization, yeast fermentation, filter-press separation of yeast, filter press separation of calcium carbonate, carbon adsorption, Continuous Stirred Tank Reactor studies, and Rotating Biological Contactor studies. These small-scale units have no control, or very rudimental control that was either built in-house (e.g. for control of $\mathrm{pH}$ in neutralization processes), or control units that were supplied by the vendors for the specific equipment (e.g. for control of small-scale bioreactors). The pilot-scale equipment of the lab consists of a single-phase evaporator with an after-condenser, a shell and tube heat exchanger, a fluid-flow set-up for pressure-drop measurement, a distillation column, an absorption column, a plate-andframe heat exchanger, and a 15-litre bioreactor. Five of these devices have been included in the process management set-up that is described in the next section.

The lab is primarily used by Mohawk College students in the three-year Chemical Engineering Technology Advanced Diploma and by McMaster University students 
in the four-year Process Automation Technology Bachelor of Technology. Because of the focus of both programs in experiential hands-on learning and the anticipated placement of graduates close to the production line or the engineering field, it was decided in summer of 2004 to invest in an industrial-type process monitoring and management system for the lab. A partnership was established with Lakeside Process Controls Ltd., the local business partner of Emerson Process Management, for the commissioning of the equipment.

\section{AUTOMATED SYSTEMS OVERVIEW}

The Emerson DeltaV process management system was selected for managing five of the pilot-scale processes of the lab: evaporator, shell-and-tube heat exchanger, fluid flow, distillation, and plate-and-frame heat exchanger. This system works in conjunction with a series of field devices that are either measurement tools, such as temperature and pressure sensors/transmitters, or final control devices, such as control valves used to increase/decrease flow and pressure. It is part of a process automation system that allows students to act as operators and control flows, steam pressures and temperatures all from PC workstations. Also, temperature and pressure profiles of the individual operations are returned to the workstation and read directly on the computer screen or HMI (Human Machine Interface). When programmed, this same information is directly exported to tables and charts to provide a seamless data stream. DeltaV is fully digital and relies on interoperable bus technology. The DeltaV system is a highly recognized, automation software system that has been awarded for its advances in process control and alarm management [2]. The details of the instrumentation and control in each of the lab processes follow.

\subsection{Instrumentation Details of Individual Processes}

The shell and tube heat exchanger is a single pass one with no buffles on the shell side. Steam, at atmospheric pressure, flows in the $12-\mathrm{cm}$ glass shell in parallel to municipal water that flows in three 0.5-in copper tubes. RTDs are used to monitor the incoming and outgoing temperatures of steam and water. Two temperature transmitters, one for both water temperatures and one for the steam temperatures, are used for displaying information on the experiment and for transferring information to the DCS. A magnetic flow meter is used for monitoring the water flow rate and a control valve is used for controlling the water flow rate through a closed feedback loop. There is no control of the steam flow rate, other than a manual valve at the process's inlet.
The plate-and-frame heat exchanger set-up is similar to that of the shell-and-tube heat exchanger. The exchanger consists of ten 6 -in by 6 -in plates used to transfer energy between hot water and cold water. Thermocouples are used to monitor incoming and outgoing temperatures of both cold and hot water. Two transmitters, one for the cold water temperatures and one for the hot water ones, are used for displaying information and transferring to the DCS. The flow rates of both cold and hot water are monitored using vortex flow meters and controlled using control valves and a feedback loop.

The fluid flow apparatus consist of a number of parallel lines used to measure pressure variation in a flow system. Line one contains an orifice, line two a venturi, line three a smooth and a sharp $360^{\circ}$ bend, line four an expansion and a long straight pipe segment, and line five a globe and a gate valve. Water runs in the system in a closed loop, being fed to the lines by a pump. The flow rate is adjusted through a manual rotameter and manual valves are used to direct the flow to one of the lines. Pressure transmitters are installed for monitoring the pressure along each line. To reduce the capital cost, the first two lines share a set of five pressure transmitters, and the final three lines share two differential pressure transmitters. A series of magnetic solenoid valves are used to select the line whose pressure taps connect to the corresponding pressure transmitters. There is no control in the system.

The distillation column has a capacity of 200 litres and eight trays. It performs batch distillation of cyclohexane and toluene at atmospheric pressure, using steam as the heating source. Thermocouples are used to record the temperature at each tray and in the column's pot. One transmitter is used for the pot's temperature, while the rest of the signals are fed to the DCS in analog form. A pressure transmitter and a control valve are used in the steam line to control, through a feedback loop, steam pressure; hence, rate of evaporation of the pot.

The evaporator has a capacity of approximately 40 liters and uses steam as the heat source. It includes a condenser and an after-condenser for reducing the system's pressure to below atmospheric. A number of thermocouples are used for monitoring temperature during the evaporation process. These are fed to the DCS in analog form. Several solenoid valves are used for controlling water flow, steam flow to the after-condenser, and condensate outflow. Finally, a pressure transmitter is utilized for monitoring the evaporator's pressure, while a differential pressure transmitter helps identify the level of "feed solution" in the evaporator. Similar to the distillation unit, a pressure transmitter and a control valve are used in the steam line to control, through a feedback loop, steam pressure; hence, the rate of evaporation. A rudimentary diagram of the process can be seen in Figure 1 in the next section. 


\subsection{Automation System Design and Connectivity}

The DeltaV is a control system capable of handling several inputs and outputs of different kinds. In this lab, the inputs are analog from certain thermocouples and digital using FOUNDATION ${ }^{\mathrm{TM}}$ fieldbus from all transmitters. FOUNDATION fieldbus is also used for connection to all the pneumatic control valves. However, the magnetic solenoid valves are connected and controlled using the AS-i network (Actuator Sensor Interface), as they require significant power for operating.

All user input and interaction with the process is accomplished through PC workstations. There are five PC workstations, one for each automated experiment. These are connected to the DeltaV DCS, through standard Ethernet cabling and hubs. Following customary industrial practice, this Local Access Network (LAN) is isolated from the outside world to ensure the integrity of the processes. To further ensure the continuous operation of the facility, there is redundancy built in. There are two identical DeltaV control consoles and each workstation is connected to both. Following the spatial arrangement of the processes, one controller normally operates the plateand-frame heat exchanger and the distillation column, while the other controls the evaporator, the fluid flow, and the shell-and-tube heat exchanger. However, in case of an emergency, either controller can manage all processes. For safety reasons, the control console closer to the distillation column must be intrinsically safe (i.e. no possibility of sparks), as there is the slightest of possibility of having explosive vapours in the area immediately surrounding the column. Another advantage of this control network is that all processes can be controlled from any workstation, which has proven advantageous in cases of unexpected hardware failures.

\subsection{Workstation roles and functionality}

Each workstation can be used in three levels: as an operator station to monitor and control operations; as a programming terminal to work with the DeltaV software at many levels from generating HMIs to writing code around the parameters to be controlled; and as an administrative station, to set up security, allow the exchange of information between computers, etc. Students are permitted to access only the operator level where they primarily interact with the process through the custom-built Human-Machine Interface (HMI). The HMI of the evaporator is shown in Figure 1, as an example.

Through the operator station, students can also view historical trends of all parameters, including controlled parameters, their set points and the value of the corresponding manipulated variables (e.g. \% of valve open). The settings of all control loops and a log of any process changes made is also available for review. Students also have to respond to alarms that may be created by process conditions.

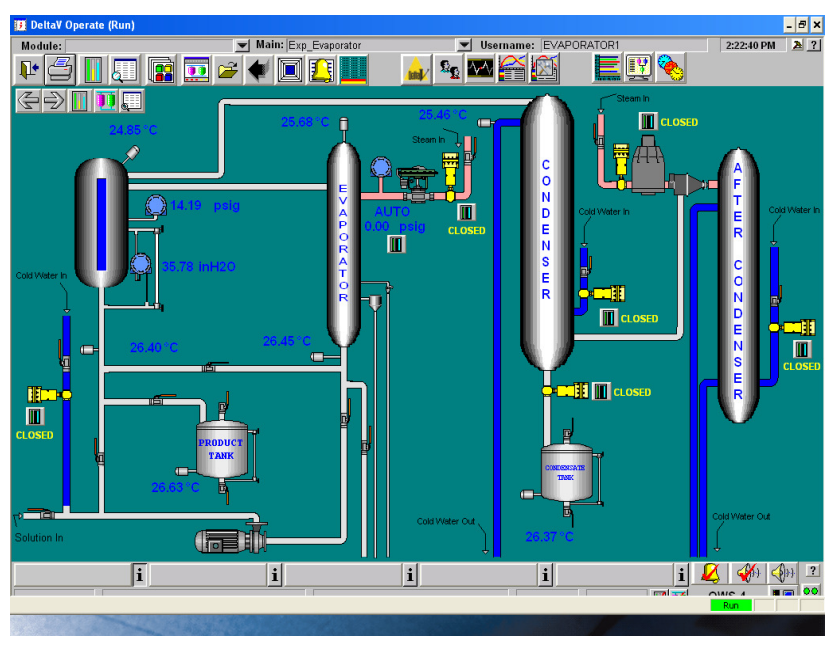

Fig. 1. Evaporator HMI

\section{BENEFITS TO STUDENTS}

Students of the two Programs that use the space, have weekly two-and-half hour labs for two terms of studies (24 labs in total). During this time period, they learn all the skills customarily associated with unit operations labs while operating both traditional/manual and automated processes. Their exposure to the automated part of the lab offers them a multitude of benefits, as outlined below.

Students become familiar with field devices. They see and experience the difference between solenoid and control valves and they understand the role electrical power and compressed air play in either valve. The difference between pressure transmitters and differential pressure transmitters is highlighted and how to differentiate between them becomes obvious. Several types of flow measuring devices are also used: automated magnetic and vortex flow meters, along with nonautomated rotameters, orifices, and venturi tubes. Also, final measuring elements (thermocouples and RTDs) are differentiated from sensors and transmitters that interpret the final element's signal and transfer it to the DCS. This is a wealth of applied instrumentation knowledge, valuable to any practicing field engineer or technologist. For the Process Automation Technology students, in particular, this builds upon an instrumentation course they had earlier in the curriculum.

The use of the workstations simulates a real-life environment where operators set process variables, monitor the process, respond to changes and alarm events. The existence of the alarms and some safety interlocks sets the framework for an elaboration on process safety, 
its critical importance and its implementation in the automation set-up. For example, in the evaporator and the distillation column, steam valves cannot be opened without first turning on cold water flows to ensure proper cooling of the process. On a different level, during operation, the inability to achieve certain process parameters, reminds students that proper control requires thoughtful design. For example, there are significant oscillations around some steam pressures because of improper valve sizing, whereas high water flow rates in the heat exchangers cannot be achieved because of inadequate water supply.

Most importantly, students experience first-hand an automation paradigm. They track the cabling while the flow of information to and from the DCS is discussed with their instructors. Differences between analog and digital communication are explained and the advantages of each are highlighted. The need for redundancy becomes clear when instructors "accidentally" disconnect the LAN to the working DCS, but the system keeps operating. Then, the applicability of different industrial networks for certain applications is experienced hands-on. Errors resulting from improper installations are also demonstrated, as a few non-critical thermocouples in the evaporator are wired with the wrong polarity, causing unrealistic results and an excellent opportunity for discussion. Finally, during their last lab session, students are asked to work in groups and produce a detailed Piping and Instrumentation Diagram (P\&ID) for one of the automated experiments. They draw on their knowledge of instrumentation from a previous class and on the supplied ISA-5.1 "Instrumentation Symbols and Identification" standard to produce a P\&ID that includes the automation aspects of the process. This has proven to be a rewarding exercise, as it solidifies their knowledge of instrumentation, the process monitoring devices, and the control set-up.

\section{CONCLUSION AND FUTURE WORK}

The automation of certain processes in the Mohawk College's Chemical Engineering lab in the summer of 2004 was a pioneer approach to bridging the gap between academic curricula and industrial practice and reality. As outlined in this paper, the automation offers a variety of benefits to students. These benefits are certainly more pronounced for the Process Automation Technology Bachelor of Technology students, who will likely be involved in designing, selecting, and setting-up automation in their post-graduation future and, through this lab, get their first exposure to an industrial-like Distributed Control System installation. Still though, the skills and experience gained are paramount for any Chemical Engineering student. Even though the lab experiments are not directly related with automation, the installation intrigues students and provides instructors with stimuli to introduce instrumentation, control, and industrial network aspects in the unit operation labs. This cross-curricula approach that is supported by handd-on experience has proven to be effective, especially when combined with courses on the automation subjects.

In the future, we plan to enhance the student experience by letting them fully exploit the possibilities of the process operator: use the historian and discuss the control parameters. There are also thoughts of introducing asset management software on certain units (pumps and control valves) for predicting the life-cycle of devices.

\section{ACKNOWLEDGEMENTS}

The authors would like to thank Bill Rolfe, lab manager at Mohawk College for his assistance in settingup the lab automation and for maintaining it ever since and Lakeside Process Control Inc. for their gracious contributions of equipment and personnel for the lab automation project.

\section{References}

[1] Ivan Castillo and Thomas F. Edgar, "Using a Distributed Control System (DCS) for distillation column control in an undergraduate unit operations laboratory", in Proc. of the American Control Conference (St. Louis, MO, 10-12 June 2009), pp. 2744-2748, 2009

[2] Emerson Electrical Inc. "Improve automation efficiency and performance with new features in DeltaV 13.0" retrieved on April 30, 2015 from http://www2.emersonprocess.com /en-us/brands/deltav/pages/index.aspx

[3] Ryan Hamilton, Anna Casasús-Zambrana, Spyros Svoronos, and Ben Koopman, "An inexpensive method for the automation of biomass measurement in lab-scale bioreactors", Journal of Laboratory Automation, Vol. 10, no. 5 pp. $315-318,2005$

[4] R. S. Kapner and R. O’Brien, "Microcomputer applications in the traditional unit operations laboratory" Computers in Education, Vol. 7, no. 3, pp. 7-10, 1987

[5] Douglas M. Perkins, David A. Bruce, Charles A. Gooding, and Justin T. Butler, "An automated distillation column for the unit operations laboratory" Chemical Engineering Education, Vol. 39, no. 2, pp. 104-108, 2005

[6] Atanas Serbezov, Ronald Antigue and Ron Knecht, "Bridging the gap between academia and Industry: RoseHulman Institute of Technology unit operations laboratory", in Proc. of the American Control Conference (St. Louis, MO, 10-12 June 2009), pp. 2735-2740, 2009 